\title{
Water-only fasting and an exclusively plant foods diet in the management of stage Illa, low-grade follicular lymphoma
}

\author{
Alan C Goldhamer, Michael Klaper, Afsoon Foorohar, Toshia R Myers
}

Department of Nutritional Medicine, TrueNorth Health Center, Santa Rosa, California, USA

\section{Correspondence to Dr Alan C Goldhamer, dracg@comcast.net}

Accepted 16 November 2015

\section{SUMMARY}

Follicular lymphoma (FL), the second most common nonHodgkin's lymphoma (NHL), is well characterised by a classic histological appearance and an indolent course. Current treatment protocols for $\mathrm{FL}$ range from close observation to immunotherapy, chemotherapy and/or radiotherapies. We report the case of a 42-year-old woman diagnosed by excisional biopsy with stage IIla, grade $1 \mathrm{FL}$. In addition to close observation, the patient underwent a medically supervised, 21-day water-only fast after which enlarged lymph nodes were substantially reduced in size. The patient then consumed a diet of minimally processed plant foods free of added sugar, oil and salt (SOS), and has remained on the diet since leaving the residential facility. At 6 and 9-month followup visits, the patient's lymph nodes were non-palpable and she remained asymptomatic. This case establishes a basis for further studies evaluating water-only fasting and a plant foods, SOS-free diet as a treatment protocol for FL.

\section{BACKGROUND}

Follicular lymphoma (FL), the second most common non-Hodgkin's lymphoma (NHL), is characterised by a classic histological appearance, an indolent course and an incurable nature. ${ }^{1}{ }^{2}$ Current treatment protocols for FL include close observation or immunotherapy when patients are asymptomatic, and immunotherapies, chemotherapies and/or radiotherapies during symptomatic periods. $^{2}{ }^{3}$ Although patients with FL have high therapeutic response rates, they can also experience high rates of relapse and a reduction in asymptomatic periods over time. ${ }^{4}{ }^{5}$ Therefore, treatment options that can manage FL symptoms and maintain asymptomatic periods with low toxicity and cost would be beneficial. Despite evidence supporting the potential role of diet in outcome survival in $\mathrm{FL}^{6}$ and various other cancers, ${ }^{6-13}$ dietary modifications are not yet a standard part of treatment protocols. The case reported here establishes a basis for further studies evaluating water-only fasting and a minimally processed plant foods diet free of added sugar, oil and salt (SOS) in the treatment and management of FL cases.

\section{CASE PRESENTATION}

On 23 June 2014, a 42-year-old woman presented to her primary care provider with a palpable mass in her right inguinal region. She was otherwise asymptomatic, denying symptoms such as fatigue, weight changes or pallor. The patient consumed the Standard American Diet, ${ }^{14}$ weighed $81.6 \mathrm{~kg}$ and had a body mass index (BMI) of $29.4 \mathrm{~kg} / \mathrm{m}^{2}$ (table 1). She had never smoked tobacco or used illicit drugs, and drank $<3$ alcoholic drinks/week. The patient's family history revealed that her father passed away from late stage cancer of undetermined origin and that distant paternal relatives had a history of renal cancer, but family history did not reveal incidence of blood or lymph cancers.

On presentation to her primary care physician, the patient was immediately sent for a CT scan and various serological tests, including a complete blood count (CBC). The CT scan obtained on 23 June 2014 revealed bilateral enlargement of the inguinal lymph nodes reflecting a notable change compared to a scan on 29 October 2012, which was obtained for an unrelated medical visit. The largest node in the right inguinal region measured $45 \times 20 \mathrm{~mm}$ (figure 1) in the transaxial long and short axis, and the largest on the left side measured $21 \times 15 \mathrm{~mm}$ (figure 2). Lymph nodes at iliac chains and pelvic sidewalls were not enlarged. There was no evidence of free fluid in the patient's pelvis and the CBC was unremarkable. On 11 July 2014, the patient underwent a surgical excisional biopsy of the left inguinal lymph node, which confirmed the diagnosis of low-grade (grade 1 of 3) follicular centre cell lymphoma. Flow cytometry showed CD-positive monotypic B cells making up 15\% of the specimen in a polyclonal B-cell background, without identification of large cell components or Burkitt's lymphoma. A chest CT scan on 15 July

Table 1 Weight and BMI

\begin{tabular}{llllll}
\hline & Diagnosis & Start of fast & End of fast & 6-Month follow-up & 9-Month follow-up \\
\hline Weight $(\mathrm{kg})$ & 81.6 & 78.0 & 68.9 & 63.0 & 59.9 \\
BMI $\left(\mathrm{kg} / \mathrm{m}^{2}\right)$ & 29.40 & 28.62 & 25.29 & 23.13 & 21.96 \\
\hline BMI, body mass index. & & &
\end{tabular}


Figure $1 \mathrm{CT}$ images of right inguinal region. (A) Before and (B) after water-only fast.
A

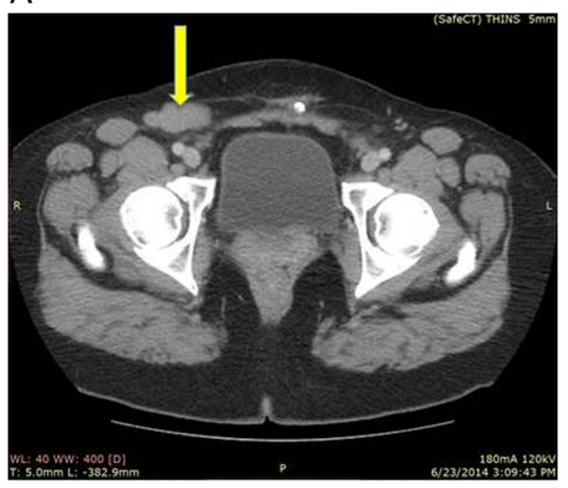

B

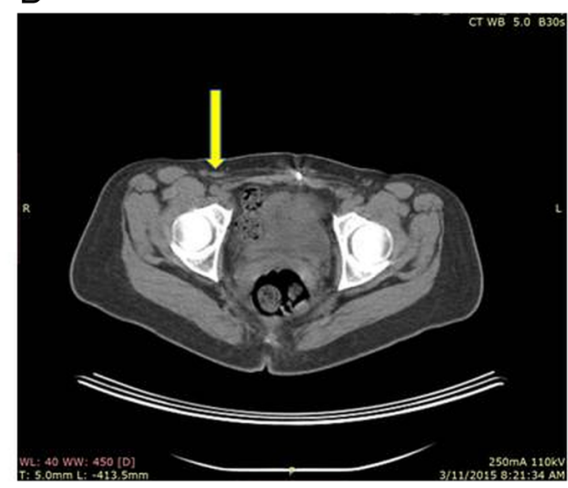

2014 revealed asymmetric enlarged lymph nodes in the right axilla, the largest measuring $28 \times 19 \mathrm{~mm}$. On the basis of the diagnosis of stage IIIa, grade $1 \mathrm{FL}$ with an unremarkable $\mathrm{CBC}$, the patient was advised to have observational follow-ups at 3-month intervals. The patient then contacted TrueNorth Health (TNH) with an expressed interest in medically supervised, water-only fasting and was determined an appropriate candidate based on the presence of adequate nutrient and electrolyte reserves, normal creatinine and haemoglobin levels, a normal glomerular filtration rate and no contraindicating conditions.

\section{DIAGNOSIS}

Diagnosis of stage IIIA, grade $1 \mathrm{FL}$ was established by excisional biopsy, according to the diagnostic criteria outlined by the 2008 WHO classification of lymphomas. ${ }^{15}$

\section{TREATMENT}

For over 30 years, TNH has safely implemented dietary modifications, including water-only fasting and an exclusively plant foods, SOS-free diet, to restore and maintain health in human patients. Water-only fasting is the complete abstinence of all foods and beverages with the exception of water. ${ }^{16}$ It is our experience that extended water-only fasting is superior to intermittent fasting in the treatment of chronic health conditions. The health promoting biological changes that occur during fasting include promotion of ketogenesis, decreased oxidative stress and inflammation, and increased stress resistance, lipolysis and autophagy. ${ }^{16}{ }^{17}$ Fasting also modulates levels of hormones and growth factors, which in turn decreases rates of cellular proliferation and increases rates of apoptosis. ${ }^{17}$ Therefore, it is thought—and emerging research is demonstrating — that fasting could potentially prevent and treat various health conditions, including some malignancies. ${ }^{17}$

Prior to arriving at $\mathrm{TNH}$, the patient began an exclusively plant foods, SOS-free diet. On 3 November 2014, the patient began to fast by completely abstaining from all foods and beverages with the exception of distilled water ad libitum (minimum of 40 ounces daily) for a period of 21 days. During the water-only fast, the patient's activities were restricted because even moderate activity doubles energy use. We have further observed, during the supervision of over 15000 fasting patients, that restricted activity also minimises the frequency of orthostatic hypotension, arrhythmia, dehydration and electrolyte disturbances-side effects reported by others who have encouraged unrestricted activity during fasting. ${ }^{16}$ The patient was allowed to engage in quiet activities (eg, reading, listening to music and watching instructional videos), participate in educational classes, and attend medical and psychological consultations. The patient's weight, blood pressure and vital signs were monitored on a twice daily basis.

The water-only fasting period was followed by 10 days of supervised refeeding beginning with the consumption of 12 ounces of unsalted vegetable broth every $3 \mathrm{~h}$ for $9 \mathrm{~h}$ on the first day. On the second day, the patient consumed 12 ounces of juice made from fresh, raw fruits and vegetables, every $3 \mathrm{~h}$ for $9 \mathrm{~h}$. On the third day, the patient consumed a diet of fresh, raw fruits and vegetables and was instructed to eat until comfortably full. Following this transitional period, a diet of minimally processed plant foods, including fresh, raw fruits and vegetables, steamed and baked vegetables, whole grains and legumes, and approximately 1 ounce/day of raw unsalted nuts and seeds, was introduced. Cooked meals were prepared with recipes that met specific guidelines ${ }^{18}$ and excluded all meat, fish, fowl, eggs and
Figure 2 CT images of left inguinal region. (A) Before and (B) after water-only fast.
A

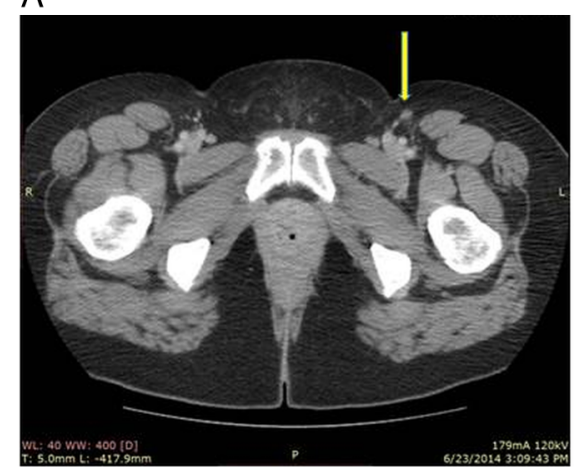

B

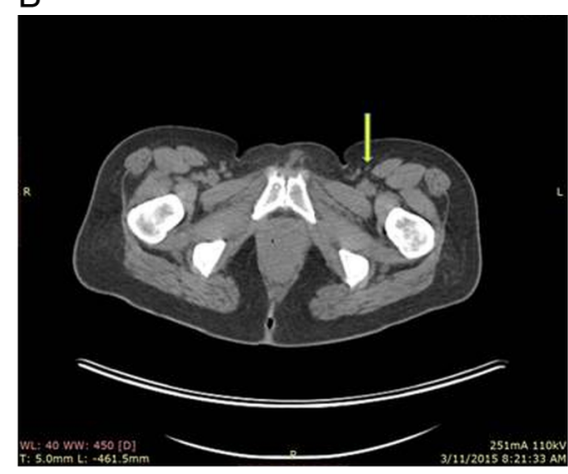


- In 2012, I had a hysterectomy and bladder suspension surgery, and ever since then my pelvic area had been sensitive and tender, so I would periodically massage it. Two years later, as I was doing this, I felt something hard in my right abdominal area. I was not sure if it was scar tissue or something else, so I made a doctor's appointment right away.

- In 2014, my doctor examined me and then asked about my family's medical history. When I told her that my dad had died from cancer, she immediately called the imaging department to request an emergency CT scan. The day after my scan, I received a call from my doctor asking me to come in to review the results. She told me that the scan had revealed tumours on both sides of my abdominal area, and that she needed to refer me to an oncologist.

- The oncologist my doctor referred me to was very kind and open to my request of wanting to avoid conventional treatment. He told me that my cancer was a non-Hodgkin's, slow growth, follicular lymphoma, and requested more tests and biopsies (one of the scans of my chest also revealed other small tumours under my right armpit). The doctor then explained that having found tumours on both the upper and lower parts of my body, and on both sides of my body, elevated the cancer to Stage III.

- His recommendation was not to treat the tumours with chemotherapy or radiation. He said the chemo would cause more damage than good and that my disease was not curable. His only course of action was to monitor me every 3 months. The first thing I did after I left my oncologist's office in 2014, was to contact Dr Klaper. After reviewing my test results, Dr Klaper said, "You are a good candidate for fasting." I checked into TrueNorth and was scheduled to do a water-only fast for 14 days. During my fast I met a veteran faster who shared with me her story of healing. After hearing about her progress, I decided to ask about fasting for a longer period. I was on my tenth day of fasting, and during morning rounds I asked Dr Klaper if it would be okay for me to extend my fast. All of my laboratory results as well as my mind, body and spirit were aligned, and he gave me the thumbs-up! I ended up extending my fast to 21 days.

- The first 3 days of water fasting, as the doctors will tell you, can be the most challenging, but after the third day it becomes easier. I was fortunate not to have experienced any severe symptoms (nausea, dizziness, fatigue). I was energetic and focused on my healing, and I listened to my body and rested when I needed to. The doctors visited me twice a day to check my vitals and to answer any questions or concerns I had. Once a week I had a blood test, and one thing that I found extremely helpful was that in each room's library of videos there was a video by Dr Klaper explaining how to read blood test results. I felt safe and found comfort in knowing that the doctors and interns were always available to answer my questions.

- Each patient at TrueNorth is given guidance on refeeding. My fast was broken systematically as solid food was slowly introduced. My first meal was vegetable broth (it was quite tasty and satisfying), and a day or two later, I had my first vegetable juice, which took me about 2-3 h to drink. I thought I would be extremely hungry, but the flavours of the juice were intense. I could now taste each individual vegetable because my taste buds had been cleaned out and reset. I consumed only vegetable juice for the next 6 days before I began to gradually add in raw vegetables. I was well monitored from the day I arrived to the day I left.

- I was on a roll, and I felt happier than I had ever been in my life. I knew that great things were happening inside my body, and that I was doing the right thing for me and my family. All I could think of was my kids and how they needed me to stick around longer than the expiration date traditional medicine had given me: 20 more years. I would be 62 by then, and I certainly did not want to die the day after I retired. My family was my inspiration, my strength and my motivation. Every day during the fast I would wake up thankful for another day, feeling happy that I was giving my body the needed time to heal. My fast at TrueNorth was one of the most life-changing and awe-inspiring experiences in my life. My water fast led to incredible reductions in the size of my tumours: When originally examined, the right inguinal node measured $4.5 \times 2.0 \mathrm{~cm}$, and now $2.6 \times 0.3 \mathrm{~cm}$. My left inguinal lymph node measured $2.1 \times 1.5 \mathrm{~cm}$, and now $1.1 \times 0.2 \mathrm{~cm}$. My right axillary node measured $2.8 \times 1.9 \mathrm{~cm}$, and now $1.5 \times 1.0 \mathrm{~cm}$. These results are truly amazing. My oncologist is pleased with the results and has asked me to keep doing what I am doing. He is very supportive of my fast and diet. Initially, he had indicated that he would observe me every 3 months, and now he says every 6 months is fine since I am making such good progress.

- My family and friends are impressed with my results and many of them have made significant changes in their diets as well. I've consulted with Dr Klaper and Dr Goldhamer since my fast, and am planning on doing a second fast later this year. Through my fasting and dietary changes my tumours have shrunk, and will continue to shrink and hopefully disappear.

- When we allow the body to rest, heal and regenerate, the results are impressive. I was fortunate to have met remarkable people, who, like me, were there to get better, and all of whom were determined to have successful outcomes. I sincerely believe that it's all about our will and positive attitude, and our determination to get well and stay healthy. My water fast changed my life, and I plan to continue to eat a wholesome, plant-based, SOS-free diet for the rest of my life. This is my medicine, this is my treatment.

dairy products, as well as added sugar, oil and salt. Bread products and other highly processed foods were also excluded. The patient was allowed to eat this specific SOS free, plant foods diet, with the exception of nuts and seeds, ad libitum. The patient was encouraged to continue consuming this diet after leaving TNH.

\section{OUTCOME AND FOLLOW-UP}

The patient did not experience any notable adverse side effects while fasting. Following the fast, her weight had reduced from
78.0 to $68.9 \mathrm{~kg}$ (table 1 ) at a rate of approximately $0.4 \mathrm{~kg} / \mathrm{day}$, her BMI had reduced from 28.62 to $25.29 \mathrm{~kg} / \mathrm{m}^{2}$ (table 1) and her $\mathrm{CBC}$ remained unremarkable. A physical examination demonstrated that the patient's enlarged lymph nodes had reduced in size. She then underwent a follow-up CT scan on 11 March 2015, and it was confirmed that the right inguinal node had reduced from $45 \times 20$ to $26 \times 3 \mathrm{~mm}$ (figure 1 ), left inguinal node had reduced from $21 \times 15$ to $11 \times 2 \mathrm{~mm}$ (figure 2 ) and the axillary node had reduced from $28 \times 19$ to $15 \times 10 \mathrm{~mm}$. The 
inguinal nodes no longer demonstrated increased fluorodeoxyglucose uptake.

On 31 January 2015, at 6-month follow-up, the patient reported strict compliance with the exclusively plant foods, SOS-free diet. Her weight was $63 \mathrm{~kg}$, BMI was $23.13 \mathrm{~kg} / \mathrm{m}^{2}$ (table 1) and the lymph nodes were non-palpable. Similarly, at 9-month follow-up, on 2 August 2015, the patient reported diet compliance, her weight and BMI had further reduced to $59.9 \mathrm{~kg}$ and $21.96 \mathrm{~kg} / \mathrm{m}^{2}$, respectively (table 1 ), and her lymph nodes remained non-palpable.

\section{DISCUSSION}

The majority of fasting research to date has been conducted on animal models, ${ }^{17}$ but there is emerging clinical data on the effectiveness of medically supervised, water-only fasting to treat a variety of human health conditions. ${ }^{16}$ For example, we have reported that fasting safely and effectively reduced blood pressure in patients with borderline hypertension ${ }^{19}$ as well as hypertension. ${ }^{20}$ There have also been clinical trials demonstrating that fasting followed by a vegetarian diet reduced inflammation and pain in rheumatoid arthritis patients. ${ }^{21}$ Additionally, fasting has been shown to reduce the side effects experienced during chemotherapy. 2223

There is currently no clinical research evaluating the effects of water-only fasting and an exclusively plant foods, SOS-free diet on FL in humans. However, there are studies reporting that alternate-day fasting reduced lymphoma in mice ${ }^{24}$ and 1 day/ week fasting reduced tumour formation in p53-deficient mice. ${ }^{25}$ One proposed mechanism by which fasting might prevent and treat cancer is by decreasing levels of insulin-like growth factor (IGF) $1 .^{26} 27$ Indeed, there is research specifically linking high IGF-1 levels to certain cancers. ${ }^{28-30}$

We have reported the case of a 42-year-old woman with stage IIIa, grade 1 FL who underwent a medically supervised, wateronly fast followed by an exclusively plant foods, SOS-free diet. Following the 21-day fast, the patient's enlarged lymph nodes were no longer palpable and CT scans confirmed that they had reduced in size. At 6 and 9-month follow-up visits, the patient reported diet compliance and her lymph nodes remained nonpalpable. These observations suggest that water-only fasting and a modified diet might be effective in the treatment and management of FL, and establishes the need for further studies. We also cannot exclude that the patient experienced a spontaneous regression, which is reported to occur in approximately $20 \%$ of low-grade NHL cases. ${ }^{31} 32$ Therefore, we will continue monitoring the patient over the coming years and report follow-up data as acquired.

\section{Learning points}

- Follicular lymphoma (FL) is an incurable, indolent form of non-Hodgkin's lymphoma.

- Current treatment protocols for FL do not include dietary modifications.

- Water-only fasting reduced enlarged lymph nodes in a case of stage IIla, grade $1 \mathrm{FL}$.

- An exclusively plant foods diet free of added sugar, oil, or salt appears to maintain the asymptomatic period in a case of stage IIla, grade $1 \mathrm{FL}$.
Competing interests None declared.

\section{Patient consent Obtained.}

Provenance and peer review Not commissioned; externally peer reviewed.

\section{REFERENCES}

1 Ansell SM. Non-Hodgkin Lymphoma: diagnosis and treatment. Mayo Clin Proc 2015;90:1152-63.

2 Hiddemann W, Cheson BD. How we manage follicular lymphoma. Leukemia 2014;28:1388-95.

3 Jacobson CA, Freedman AS. Is observation dead in follicular lymphoma? Still appropriate. J Nat/ Compr Canc Netw 2015;13:367-70.

4 Ujjani C. Targeted approaches to the management of follicular lymphoma. Oncology (Williston Park) 2015;29:760-8.

5 Rueda A, Casanova M, Redondo $M$, et al. Has the time to come leave the "watch-and-wait" strategy in newly diagnosed asymptomatic follicular lymphoma patients? BMC Cancer 2012;12:210.

6 Han X, Zheng T, Foss F, et al. Vegetable and fruit intake and non-Hodgkin lymphoma survival in Connecticut women. Leuk Lymphoma 2010;51:1047-54.

7 Kwan ML, Weltzien E, Kushi LH, et al. Dietary patterns and breast cancer recurrence and survival among women with early-stage breast cancer. J Clin Oncol 2009;27:919-26.

8 Kroenke $\mathrm{CH}$, Fung TT, Hu FB, et al. Dietary patterns and survival after breast cancer diagnosis. J Clin Oncol 2005;23:9295-303.

9 Pierce JP, Stefanick ML, Flatt SW, et al. Greater survival after breast cancer in physically active women with high vegetable-fruit intake regardless of obesity. J Clin Oncol 2007;25:2345-51.

10 Rock CL, Demark-Wahnefried W. Nutrition and survival after the diagnosis of breast cancer: a review of the evidence. J Clin Oncol 2002;20:3302-16.

11 Goodman MT, Kolonel LN, Wilkens LR, et al. Dietary factors in lung cancer prognosis. Eur J Cancer 1992;28:495-501.

12 Palli D, Russo A, Saieva C, et al. Dietary and familial determinants of 10-year survival among patients with gastric carcinoma. Cancer 2000;89:1205-13.

13 Sandoval $M$, Font $R$, Manos $M$, et al. The role of vegetable and fruit consumption and other habits on survival following the diagnosis of oral cancer: a prospective study in Spain. Int J Oral Maxillofac Surg 2009;38:31-9.

14 Grotto D, Zied E. The Standard American Diet and its relationship to the health status of Americans. Nutr Clin Pract 2010;25:603-12.

15 Jaffe ES. The 2008 WHO classification of lymphomas: implications for clinical practice and translational research. Hematology Am Soc Hematol Educ Program 2009:523-31.

16 Pizzorno JM, Murray T. Textbook of natural medicine. 4 edn. Elsevier Churchill Livingstone, 2013.

17 Longo VD, Mattson MP. Fasting: molecular mechanisms and clinical applications. Cell Metab 2014;19:181-92.

18 Goldhamer A. The health promoting cookbook. Summertown, TN: Book Publishing Company, 1996.

19 Goldhamer AC, Lisle DJ, Sultana P, et al. Medically supervised water-only fasting in the treatment of borderline hypertension. J Altern Complement Med 2002;8:643-50.

20 Goldhamer A, Lisle D, Parpia B, et al. Medically supervised water-only fasting in the treatment of hypertension. J Manipulative Physiol Ther 2001;24:335-9.

21 Muller H, de Toledo FW, Resch KL. Fasting followed by vegetarian diet in patients with rheumatoid arthritis: a systematic review. Scand J Rheumatol 2001;30:1-10.

22 Safdie FM, Dorff T, Quinn D, et al. Fasting and cancer treatment in humans: a case series report. Aging (Albany NY) 2009;1:988-1007.

23 Raffaghello L, Safdie F, Bianchi G, et al. Fasting and differential chemotherapy protection in patients. Cell Cycle 2010;9:4474-6.

24 Descamps 0 , Riondel J, Ducros V, et al. Mitochondrial production of reactive oxygen species and incidence of age-associated lymphoma in OF1 mice: effect of alternate-day fasting. Mech Ageing Dev 2005;126:1185-91.

25 Berrigan D, Perkins SN, Haines DC, et al. Adult-onset calorie restriction and fasting delay spontaneous tumorigenesis in p53-deficient mice. Carcinogenesis 2002;23:817-22.

26 Cheng CW, Adams GB, Perin L, et al. Prolonged fasting reduces IGF-1/PKA to promote hematopoietic-stem-cell-based regeneration and reverse immunosuppression. Cell Stem Cell 2014;14:810-23.

27 Lee C, Safdie FM, Raffaghello L, et al. Reduced levels of IGF-I mediate differential protection of normal and cancer cells in response to fasting and improve chemotherapeutic index. Cancer Res 2010;70:1564-72.

28 Brahmkhatri VP, Prasanna C, Atreya HS. Insulin-like growth factor system in cancer: novel targeted therapies. Biomed Res Int 2015;2015:538019. 
29 Zielinska HA, Bahl A, Holly JM, et al. Epithelial-to-mesenchymal transition in breast cancer: a role for insulin-like growth factor I and insulin-like growth factor-binding protein 3? Breast Cancer (Dove Med Press) 2015;7:9-19.

30 Cao Y, Nimptsch K, Shui IM, et al. Prediagnostic plasma IGFBP-1, IGF-1 and risk of prostate cancer. Int J Cancer 2015;136:2418-26.
31 Horning SJ, Rosenberg SA. The natural history of initially untreated low-grade non-Hodgkin's lymphoma. N Engl J Med 1984;311:1471-5.

32 Drobyski WR, Qazi R. Spontaneous regression in non-Hodgkin's lymphoma: clinical and pathogenetic considerations. Am J Hematol 1989;31:138-41.

Copyright 2015 BMJ Publishing Group. All rights reserved. For permission to reuse any of this content visit

http://group.bmj.com/group/rights-licensing/permissions.

BMJ Case Report Fellows may re-use this article for personal use and teaching without any further permission.

Become a Fellow of BMJ Case Reports today and you can:

- Submit as many cases as you like

- Enjoy fast sympathetic peer review and rapid publication of accepted articles

- Access all the published articles

- Re-use any of the published material for personal use and teaching without further permission

For information on Institutional Fellowships contact consortiasales@bmjgroup.com

Visit casereports.bmj.com for more articles like this and to become a Fellow 